\title{
Plasmonic imaging of protein interactions with single bacterial cells
}

Karan Syal,1 Wei Wang, ${ }^{2}$ Xiaonan Shan,,1,3 Shaopeng Wang, ${ }^{1,2}$ Hong-Yuan Chen², and Nongjian Tao*1-3

${ }^{1}$ Center for Biosensors and Bioelectronics, Biodesign Institute, Arizona State University, Tempe, Arizona 85287 USA.

${ }^{2}$ State Key Laboratory of Analytical Chemistry for Life Science, School of Chemistry and Chemical Engineering, Nanjing University, Nanjing 210093, China

${ }^{3}$ School of Electrical, Computer and Energy Engineering, Arizona State University, Tempe, Arizona 85287, USA.

Email:njtao@asu.edu; hychen@nju.edu.cn

*Corresponding authors.

\begin{abstract}
Quantifying the interactions of bacteria with external ligands is fundamental to the understanding of pathogenesis, antibiotic resistance, immune evasion, and mechanism of antimicrobial action. Due to inherent cell-to-cell heterogeneity in a microbial population, each bacterium interacts differently with its environment. This large variability is washed out in bulk assays, and there is a need of techniques that can quantify interactions of bacteria with ligands at the single bacterium level. In this work, we present a label-free and real-time plasmonic imaging technique to measure the binding kinetics of ligand interactions with single bacteria, and perform statistical analysis of the heterogeneity. Using the technique, we have studied interactions of antibodies with single Escherichia Coli 0157:H7 cells and demonstrated a capability of determining the binding kinetic constants of single live bacteria with ligands, and quantify heterogeneity in a microbial population.
\end{abstract}


Keywords: Biosensors, Surface Plasmon Resonance Microscopy, Single Microbial cell, Antigen-Antibody Kinetics, Analytical Chemistry, Bacterial Population Heterogeneity

\section{Introduction}

Bacteria interact with environment through their surface constituents, such as lipid bilayers, peptidoglycan layers, lipopolysaccharides (LPS), pilli, flagella and outer membrane proteins. The surfaces of bacteria act as the first line of defense against harmful external stimuli, including antibiotics(Delcour, 2009) and antimicrobial peptides,(Fantner et al., 2010; Sochacki et al., 2011) and also play crucial roles in interacting with other surfaces, including host tissues(Van Houdt and Michiels, 2005) and medical plastics,(Lower et al., 2011) to help bacterial cells attach and colonize. In order to survive in a changing environment, bacteria replicate and evolve quickly,(Carnes et al., 2010; van der Mei and Busscher, 2012) leading to diversity of different bacteria species, and variability within the same species.(van der Mei and Busscher, 2012; Woude and Bäumler, 2004) It is thus important to study and quantify the interactions of bacteria with external ligands at the single bacterium level.

The interactions of external ligands and bacteria have been studied using $e x$ situ and in situ approaches, such as fluorescence assay,(Cywes-Bentley et al., 2013) quartz crystal microbalance (QCM)(Shen et al., 2007), surface plasmon resonance (SPR)(Medina et al., 1997; Subramanian et al., 2006), microcantilevers(Longo et al., 2013) and atomic force microscope (AFM).(Fantner et al., 2010; Lower et al., 2011) The ex situ approaches include the study of reconstituted artificial membranes(Früh 
et al., 2011; Hirst et al., 2013), membrane protein embedded liposomes(Liu and Boyd, 2013), and extracted surface constituents (e.g., membrane proteins(Holden et al., 2006) and sugars(Grant et al., 2008) from bacteria. Given the complexity of the bacteria, in situ study of intact bacterial cells in their native environments are more attractive.(Lee, 2004)

Traditional studies of intact bacteria cells are largely based on bulk assays and susceptibility testing assays, using techniques such as SPR(Chiang et al., 2009; Medina et al., 1997; Subramanian et al., 2006) and disk-diffusion(Jorgensen and Ferraro, 2009). The data generated with these bulk assays are averaged over many bacteria, which wash out important variability or heterogeneity of different bacterial cells. Various imaging techniques, such as fluorescence(Cywes-Bentley et al., 2013; Sochacki et al., 2011), AFM (Fantner et al., 2010; Lower et al., 2011) and transmission electron microscopy (TEM)(Cywes-Bentley et al., 2013), and nonimaging microfluidics techniques, such as flow cytometry(Tracy et al., 2010) and micro electrophoresis(van der Mei and Busscher, 2012), have been used to study bacterial surfaces. These techniques have contributed to the understanding of bacteria, but each has disadvantages. For example, the fluorescence method requires labeling, which limits its application to only certain probe molecules and cultivable strains, and gram negative bacteria with sugars cannot be easily labeled by engineering cells.(Chang and Bertozzi, 2012) In addition, the fluorescence method is an end-point assay, which is not suitable for quantifying the kinetics of molecular binding to bacteria. TEM requires extensive sample preparations and is unsuitable for live cell analysis in aqueous solutions. AFM can operate in aqueous 
solutions, but it is usually too slow to follow fast binding of ligands with bacteria, and the scanning AFM probe may perturb the binding process. In this study, we present a plasmonic imaging technique(Huang et al., 2007; Wang et al., 2010, 2012) (Fig. 1a) to study and quantify the interactions of a single E. Coli 0157:H7 cell with an antibody, and perform statistical analysis of the bacterial heterogeneity.

E. Coli 0157:H7 is a highly virulent food borne pathogen that causes diseases, such as diarrhea, hemorrhagic colitis and hemolytic uremic syndrome.(Besser et al., 1999) Many groups have tried detecting this pathogen by several culture assays as well biosensing approaches. Several groups have used conventional SPR to detect $E$. Coli 0157:H7 by direct detection of bacterial cells binding to surface(Tawil et al., 2012; Torun et al., 2012), indirect detection of surface immobilized cells using complementary probes(Medina et al., 1997; Subramanian et al., 2006) or coupling SPR with other techniques.(Zordan et al., 2009) In this study, we focus on the binding kinetics of goat anti-E Coli 0157:H7 IgG polyclonal antibody(Ab157)(Medina et al., 1997; Subramanian et al., 2006) onto single E. Coli 0157:H7 cells. Commercial humanized antibodies are increasingly used as an alternate therapy for immune clearance of pathogens,(Casadevall et al., 2004; Cywes-Bentley et al., 2013) hence the study of antibody binding kinetics with single bacterial cells is important to elucidate their efficacy and potential as future drugs. 


\section{Materials and Methods}

Materials - Lyophilized Bacterial pellets of E. Coli 0157:H7 (ATCC43888) were purchased from Fisher Scientific. Affinity purified goat anti-E. coli 0157:H7 IgG and anti-E. coli 0145:H7 polyclonal antibodies were purchased from Kirkegaard and Perry Laboratory Inc. (Gaithersburg, MD) and suspended in 1ml PBS (1x). Secondary antibody Alexa Fluor 555 rabbit anti-goat $\operatorname{IgG}(\mathrm{H}+\mathrm{L})$ was purchased from Life Technologies (Carsbad, CA). (1-Mercapto-11-undecyl) hexa(ethylene glycol) (PEG) and Carboxyl-terminated hexa(ethylene glycol) undecane thiol (PEG-COOH) was purchased from Nanoscience Instruments (Phoenix). Other reagents were purchased from Sigma-Aldrich.

Bacteria Purification - The lyophilized bacteria was suspended in 1ml PBS (1x) and cleaned by centrifugation at the speed of $50 \mathrm{~g}$ for 1 minute to remove charcoal and collecting the supernatant containing bacteria. The supernatant was collected and washed further by pelleting bacteria in the centrifuge at the speed of $2000 \mathrm{~g}$ for 15 minutes. The pelleted bacteria was suspended in $1 \mathrm{ml}$ PBS and mixed thoroughly. Further, the above washing step was repeated three times. The final $1 \mathrm{ml}$ of bacteria in PBS solution, after 3 rounds of purification, was saved in small aliquots of $20 \mu \mathrm{l}$ and frozen at $-80^{\circ} \mathrm{C}$.

Surface Functionalization - Clean BK7 glass cover slips were coated with $1.5 \mathrm{~nm}$ chromium and $47 \mathrm{~nm}$ gold to prepare SPR chips. The chips were cleaned with deionized water and ethanol multiple times and blown dry with nitrogen gas and 
then cleaned by hydrogen flame. The cleaned chips were submerged in $1 \mathrm{mM}$ PEG/PEG-COOH ethanol solution and left overnight in dark for $24 \mathrm{hrs}$. The overnight incubated chips were taken out and cleaned with deionized water, ethanol solution multiple times and blown dry with nitrogen gas. PEG/PEG-COOH self-assembled monolayer (SAM) was deposited on each chip with this protocol.

Next, the SAM coated chip was activated with $0.5 \mathrm{ml}$ of freshly prepared mixture (1:1) of $0.1 \mathrm{M}$ NHS and $0.4 \mathrm{M}$ EDC to produce NHS ester receptors capable of binding with amino group of antibodies via an amide bond. The chip was cleaned with deionized water and blown dry with nitrogen gas. Polyclonal anti-E.Coli 0157:H7 antibody suspended in $20 \mathrm{mM}$ sodium acetate (NaOAc) $\mathrm{pH} 5.5$ at the concentration $(30 \mathrm{ug} / \mathrm{ml})$ optimized for maximum bacteria immobilization was immediately applied to NHS/EDC activated surfaces above and kept for about 60-90 mins.(Subramanian et al., 2006) The chip was again cleaned by deionized water and blown dry with nitrogen gas. This antibodies conjugated sensor chips are ready for bacteria capture on SPRM setup later.

Plasmonic imaging and Flow Setup - The plasmonic imaging setup is based on the Kretschmann configuration with a high numerical aperture objective (NA 1.49) and an inverted microscope (Olympus IX81) (Fig. 1). The sensor chip was placed on the objective lens with refractive index matching immersion oil. A $680 \mathrm{~nm}$ super luminescence diode (Qphotonics, Ann Arbor, MI) was used to excite the SPR images and a CCD camera (Pike-032B, Allied Vision Technologies, Newbuyport, MA) was used to record SPRM images. 
A FlexiPerm sample well was mounted on top of the antibody functionalized gold chip and filled with PBS (1x) buffer. The assembled gold chip was then mounted on top of the plasmonic imaging setup. The incident angle of the light beam was adjusted to the surface plasmon resonance angle, showing minimal image intensity.

Sample delivery was based on a multichannel gravity based drug perfusion system, which flew sample solutions over the immobilized bacterial cells. The flow rate was $330 \mu \mathrm{l} / \mathrm{min}$ and the transition time between different flow solutions was in the range of 1-2 seconds.

Bacteria Immobilization - An aliquot of frozen purified bacteria was thawed for 2 minutes, and then $20 \mathrm{ul}$ of bacteria were added to the sensor chip. The bacteria started to attach and immobilize onto the sensor surface via antibody binding. After about 15-20 minutes of incubation, sufficient amount of bacteria were attached onto the gold chip. 1x PBS buffer was flowed over the chip to wash out unattached bacteria from the solution, then $5 \mathrm{mg} / \mathrm{mL}$ BSA were added to the chip and incubated for 1 hour to completely block the surface and prevent non-specific adsorption of antibody.

Immunofluorescence microscopy - Following kinetics of Ab157 binding to E. Coli 0157:H7 cells, fluorescence labeled secondary antibody at a concentration of 10 $\mu \mathrm{g} / \mathrm{ml}$ was introduced for 5 minutes and washed with PBS to remove unbound secondary antibody. 
Image Collection and Processing - All plasmonic imaging videos were collected at $3.3 \mathrm{fps}$ at a pixel resolution of $640 \times 480$. We chose an appropriate exposure time to maximize image intensity at the same time avoiding over exposure. Images were subtracted from the first recorded image to remove background noises and interference patterns. Further, images were plotted in 2D frequency domain using Fast Fourier Transform (FFT) conversion, and cleaned up with a spatial band pass filter. The cleaned images were converted back from frequency domain using inverse FFT. For better visualization, images were converted to scaled color images.

Data analyses from Images - As mentioned in text, the plasmonic imaging intensity in each of the selected regions, including regions of the bacteria and bare gold chip regions, was analyzed with a MATLAB program. The plasmonic image intensity from each bacterium was reference corrected with the background bare gold regions to analyze the binding kinetics. The binding kinetics was determined with the first order kinetic equation, and $k_{a}, k_{d}$ and $K_{D}\left(=k_{a} / k_{d}\right)$ were obtained from the fitting. 


\section{Results and Discussion}

E. Coli 0157:H7 bacteria were immobilized on the sensor chip using an antiEColi 0157 (antibody Ab157), which were imaged with a plasmonic imaging setup (Fig. 1a) described in detail elsewhere.(Huang et al., 2007; Shan et al., 2012; Wang et al., 2010, 2012, 2011) Briefly, the setup is based on an inverted optical microscope (Olympus IX81) with high numerical aperture oil immersion objectives (N.A.=1.49). Light from a super-luminescence diode (Qphotonics, Ann Arbor, MI) with wavelength of $680 \mathrm{~nm}$ is directed onto the sensor chip, made of glass coverslip coated with a $47 \mathrm{~nm}$ thick gold film. The incident angle of light is tuned to excite surface plasmons, and the reflected light is imaged with the same objective together with other components, including a CCD imager.

The individual bacteria were imaged as distinct V-shaped patterns (Fig. 1c), which match well with the positions of the bacteria in the Bright-field optical image (Fig. 1b). These V-shaped patterns are caused by the scattering of surface plasmonic waves by the bacteria immobilized on the surface.(Wang et al., 2010) The distinct patterns in the plasmonic image are helpful to distinguish bacterial cells from interference patterns originated from the optical setup, and other spatial background noises on the image. 
a

d

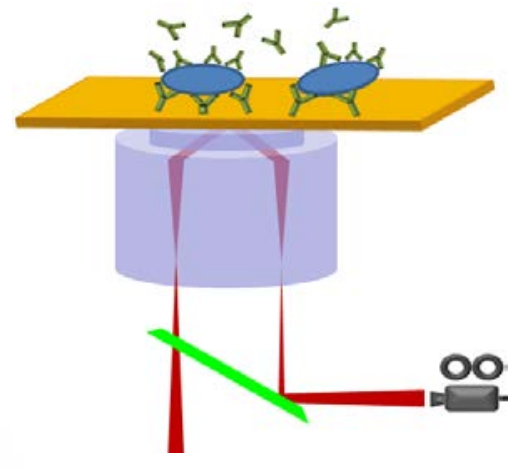

b

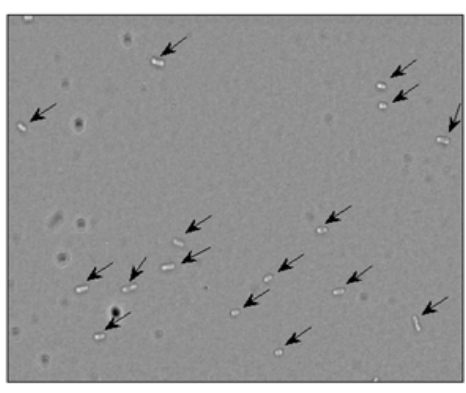

$\mathbf{e}$

C
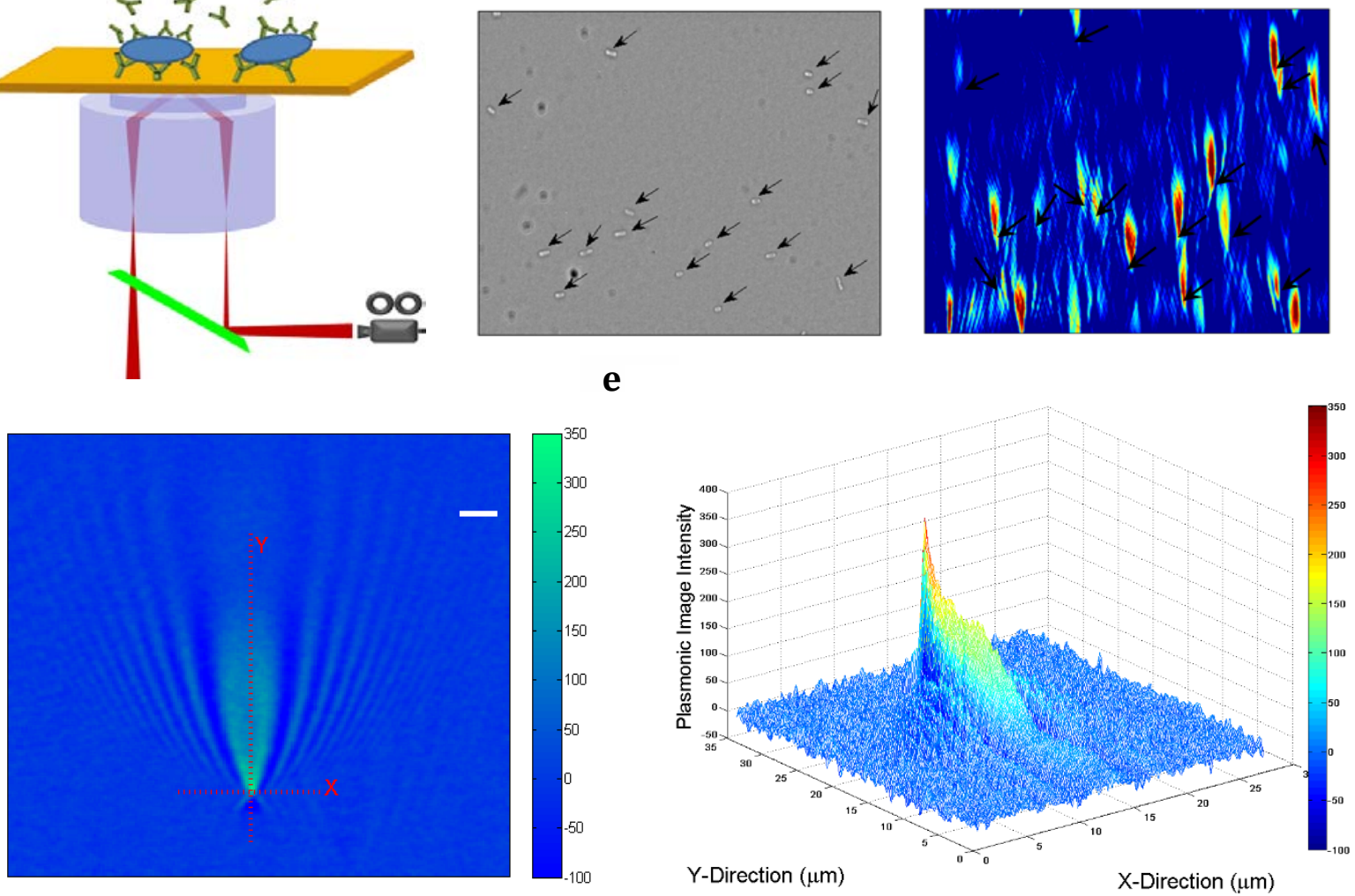

g

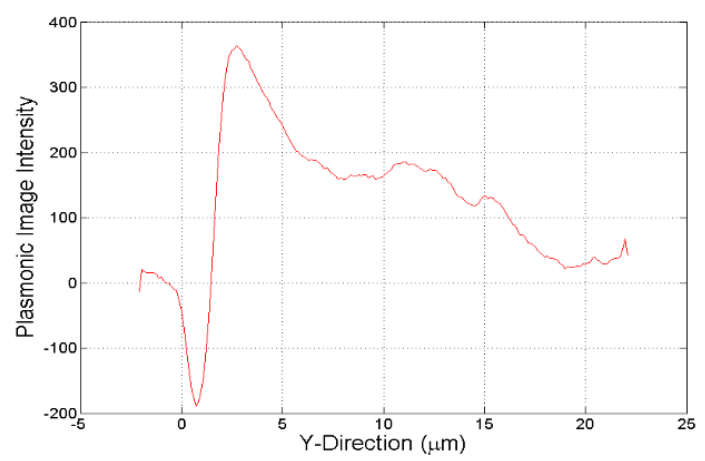

Fig. 1a) Schematic of the plasmonic imaging setup using a high numerical objective and the immobilization of bacterial cells on top of gold chip by covalently attached antibodies. b) Bright-field optical image of immobilized bacteria. c) Plasmonic image of bacteria shown as V-shaped diffraction patterns at positions of bacteria on bright field image. d) Magnified 
plasmonic image of a single bacterium showing clearly the V-shape diffraction pattern. e) 3D histogram of the bacteria in Fig. 1d. f) Profile of the V-shaped pattern along the basin of V in Fig. 4d. g) Profile of the V-shaped pattern along the middle axis in Fig. 4d. Scale bar: $2 \mu \mathrm{m}$

Figs. $1 \mathrm{~d}$ and e show more clearly one of the V-shaped patterns, and also reveal that the region of maximum intensity in each V-shaped pattern overlays one immobilized bacterial cell. The intensity profile along the basin of the $\mathrm{V}$ shaped diffraction pattern (X-direction) of bacterial cells show a full-width at half maximum (FWHM) of about $0.5 \mu \mathrm{m}$ (Fig. 1f). This FWHM is due to the optical diffraction limit of our setup.(Wang et al., 2010) The intensity profile along the plasmonic wave propagation direction (Y-direction) reveals that the intensity decays with a FWHM of about $\sim 4 \mu \mathrm{m}$ (Figure $1 \mathrm{~g}$ ). The intensity decay is due to the finite propagation length of surface plasmonic waves, which depends on the type of the metal film, and the wavelength of incident light(Wang et al., 2010). We observe similar V-shaped patterns for all immobilized bacterial cells with no major differences in the decay length and FWHM for different cells.

Using the plasmonic imaging setup, we next imaged the binding process of Ab157 antibody to the E. Coli O157:H7 cells immobilized on the surface. Fig. 2a shows a bright-field image of 4 immobilized bacterial cells. We started the binding study by initially flowing $1 x P B S$ at $330 \mu \mathrm{l} /$ min continuously over the bacterial cells for about 2 minutes. We then switched the flow to $1 \times$ PBS containing $10 \mu \mathrm{g} / \mathrm{mL}$ Ab157 to study the association of the antibody to the individual bacterial cells. After 
3 minutes, we switched the flow back to 1 xPBS in order to follow the dissociation process.

Since we are interested in the association and dissociation processes of antibody onto the bacteria, time-differential images were obtained by subtracting the first frame from the subsequent frames to show changes in the image over time. Fig. $2 \mathrm{~b} 1$ is a time-differential image captured before the introduction of solution containing antibody, which shows weak contrast of the bacteria. If the bacteria were static, then there should be no contrast in the time-differential image before antibody binding taking place. The observation of the small contrast is due to micromotions of the live bacterial cells. We will return to this later.

Upon exposure to the PBS containing the antibody, binding of the antibody to the bacteria, primarily via the 0-antigen(Park et al., 1998) on the outer membrane surfaces of the bacteria, takes place, which is revealed as an increase in the image contrast of the individual bacterial cells. Figs. $2 \mathrm{~b} 2-4$ show several time-differential plasmonic images of the association process from which detailed information of the association process can be obtained. For example, the images show the contrast increases for different cells are different, demonstrating the cell-to-cell heterogeneity that is washed out in the bulk assay. The images also show the increase in the intensity in the regions between the bacterial cells, which, as we will discuss later, is mainly due to the increase in the bulk refractive index as we switch the solution. 
Snapshots of the time-differential plasmonic images during the dissociation process are shown in Figs. 2b5-7, which were captured after switching the antibodycontaining solution back to PBS. During the dissociation phase, the image intensity of each bacterial cell decreases at a slow rate compared to the association process, which is expected as the antibodies dissociate from the bacterial cell. Like the association process, the dissociation also varies across different cells. Note also that the image intensity in the regions without bacteria returns to the baseline level quickly, which further supports the interpretation of bulk refractive index change as the origin of the intensity change in these regions.

a
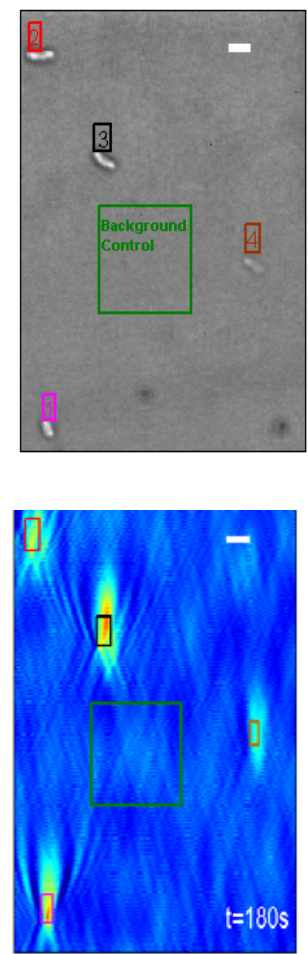

b4 b1
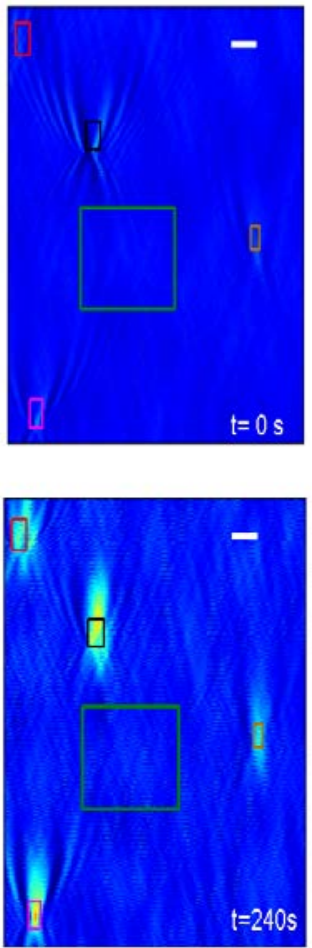

b5 b2
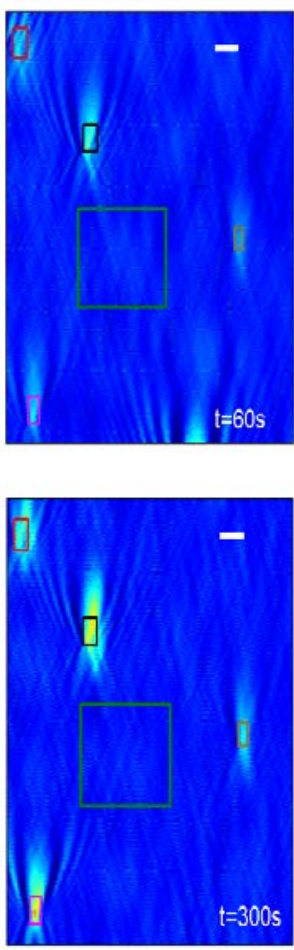

b6 b3
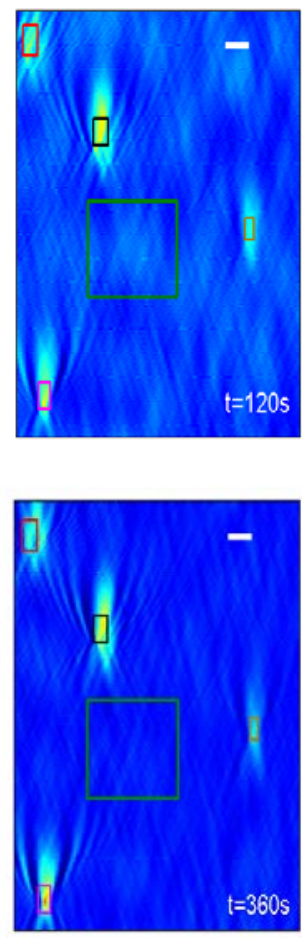

b7

Fig. 2 a) Bright-field optical image of immobilized E. Coli 0157:H7 cells. The region chosen to analyze the plasmonic image intensity of each bacterium is marked as a colored box in the 
bright-field image. Also shown is the background control region as a green box near the center of the image. b) Time-differential plasmonic images captured during different stages of association (b1-b4) and dissociation (b4-b7) processes. A complete plasmonic video of the binding kinetics is given in the supporting information. Scale bar: $2 \mu \mathrm{m}$.

The image intensity vs. time profiles obtained from the recorded image sequence provides detailed kinetic information of the antibody binding to the bacterial cells (Fig. 3). This type of plots has been widely referred to as sensorgrams, but Fig. 3 represents the first sensorgrams on single bacterial cells. The sensorgrams are "noisy", which is mainly due to micromotions of the bacteria. The bacterial cells in the present work were alive and attached to the sensor surface via relatively weak non-covalent bonds, so we observed frequent movement of the bacterial cells in the plasmonic images. Despite the micro-motions, the sensorgrams can be fit with the first order kinetics model, from which kinetic constants, including $k_{a}, k_{d}$ and $K_{D}$, for each of the bacteria cells are obtained (see Table 1). Fig. 3 also plots the image intensity vs. time profile of a region between the bacteria cells, which shows only a bulk index change with no binding kinetics curve as observed over bacterial cells. This observation indicates that non-specific binding of the antibody to the sensor surface is insignificant. 


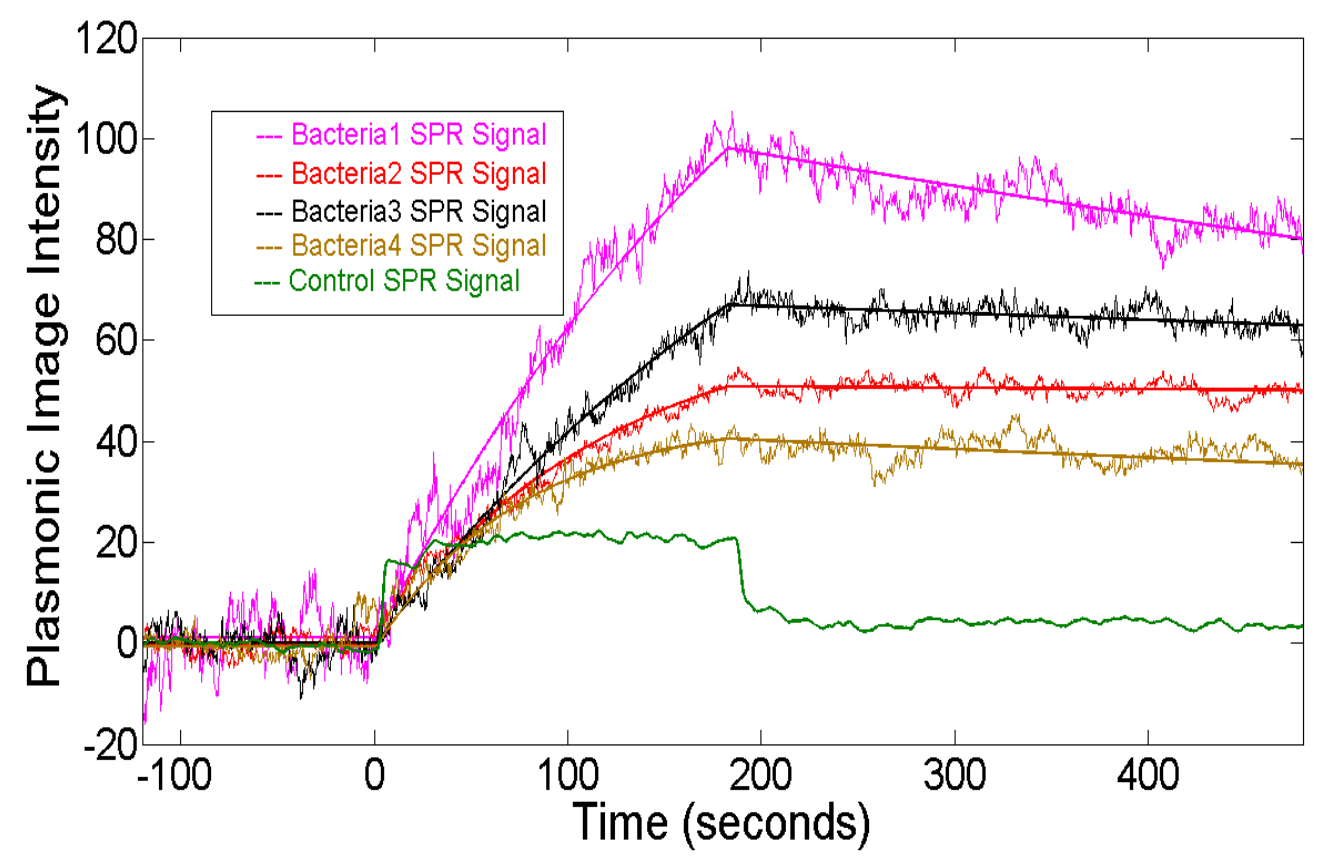

Fig. 3 Sensorgrams of single bacterial cells obtained by plotting the image intensity vs. time. Smooth solid lines are fits to the first order kinetics for different cells, allowing the determination of kinetic constants, $\mathrm{k}_{\mathrm{a}}, \mathrm{k}_{\mathrm{d}}$ and $\mathrm{K}_{\mathrm{D}}$ for each of the bacteria cells. The green line plots the sensorgram of a region without bacteria cells, showing a sudden increase and decrease as the flow is switched from PBS buffer solution to sample solution, and then back to PBS buffer, respectively.

\section{Table1 - Kinetics of individual Microbial cells}

\begin{tabular}{|c|c|c|c|c|}
\hline Bacteria & $\begin{array}{c}\text { Association rate } \\
\left(\mathrm{k}_{\mathrm{a}}\right)\left(\mathrm{M}^{-1} \mathrm{~s}^{-1}\right)\end{array}$ & $\begin{array}{c}\text { Dissociation rate }\left(\mathrm{k}_{\mathrm{d}}\right) \\
\left(\mathrm{s}^{-1}\right)\end{array}$ & $\begin{array}{c}\text { Dissociation Constant }\left(\mathrm{K}_{\mathrm{D}}\right) \\
(\mathrm{M})\end{array}$ & $\begin{array}{c}\text { Peak Plasmonic } \\
\text { Image intensity }\end{array}$ \\
\hline Bacteria 1 & $5.1 \times 10^{4}$ & $6.9 \times 10^{-4}$ & $1.3 \times 10^{-8}$ & 97.5 \\
\hline Bacteria 2 & $1.3 \times 10^{5}$ & $5.1 \times 10^{-5}$ & $4.0 \times 10^{-10}$ & 50.8 \\
\hline Bacteria 3 & $5.1 \times 10^{4}$ & $2.1 \times 10^{-4}$ & $4.1 \times 10^{-9}$ & 67.0 \\
\hline Bacteria 4 & $2.0 \times 10^{5}$ & $4.4 \times 10^{-4}$ & $2.3 \times 10^{-9}$ & 40.1 \\
\hline
\end{tabular}


To further validate the plasmonic imaging of the antibody binding to bacterial cells, we used AlexaFlor555 labeled secondary antibody to bind to the attached primary antibody. Fig. 4 shows the conventional bright field optical, timedifferential plasmonic and fluorescence images of a sensor surface covered with multiple bacteria cells. The fluorescence image (Fig. 4c) confirms that the increase in the plasmonic image intensity is due to the binding of the primary antibody to the bacterial cells. As an additional validation experiment, we used a goat anti-E. coli O145 IgG polyclonal antibody (Ab145) as a negative control. The exposure of E. Coli cells to Ab145 did not change the plasmonic image intensity (Supplemental Information - Fig. 1), indicating that the binding kinetics of Ab157 with E. Coli cells were specific.

The results discussed above demonstrate a new capability for studying the binding kinetics of single bacteria. Compared to the conventional SPR approach which studies a layer of many bacterial cells immobilized on a sensor surface,(Medina et al., 1997; Subramanian et al., 2006) the single bacteria binding kinetics analysis capability can discriminate non-specific binding taking place on the regions of the sensor surface without bacteria cells. More importantly, it opens up the possibility of detecting individual microbes in mixed communities, biofilms as well as microbe-infected patient samples. We demonstrate below kinetics analysis of multiple bacterial cells and cell-to-cell heterogeneity. 
a

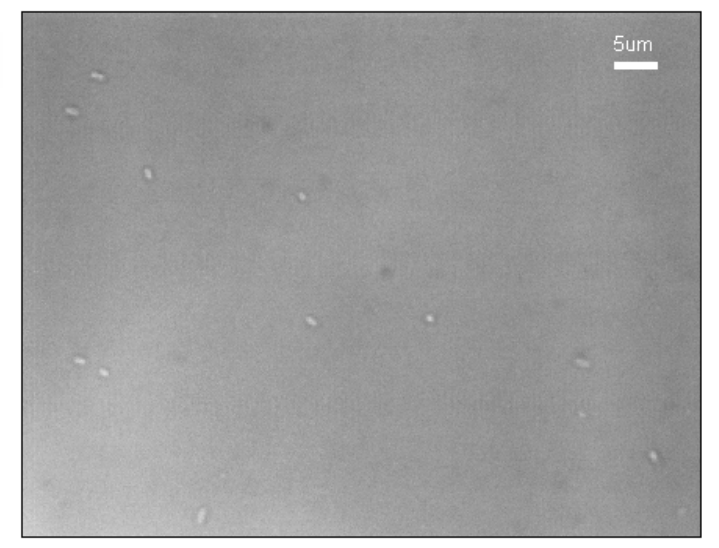

b

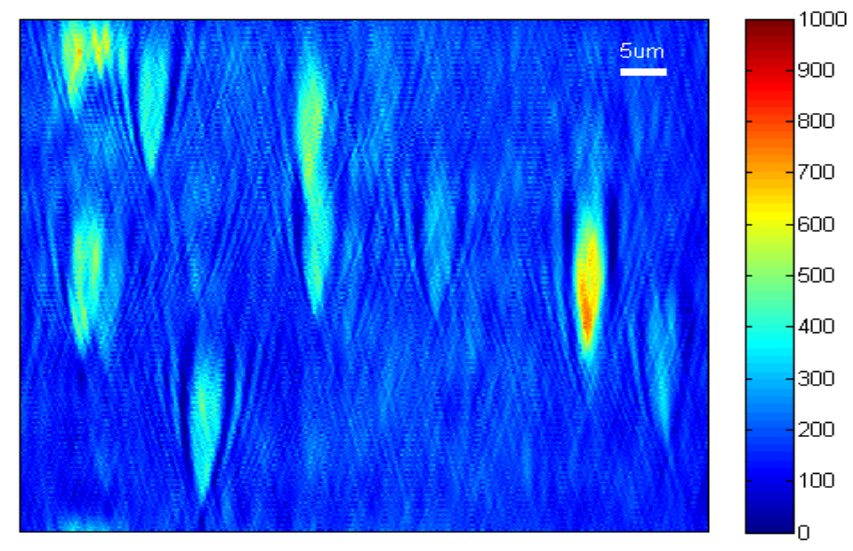

c

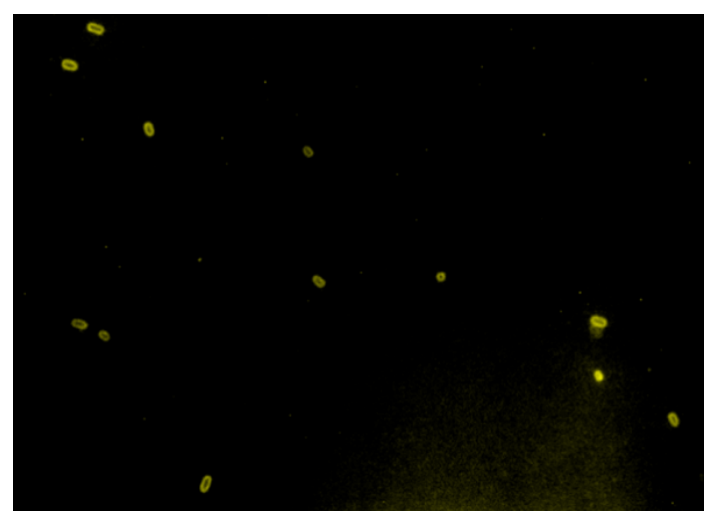

Fig 4. a) Bright-field optical image of bacteria immobilized on a gold chip. b) Timedifferential plasmonic image showing V-shape diffraction patterns corresponding to the individual bacterial cells in Fig. 4a. c) Fluorescence image after adding secondary antibody labelled with Alexaflor555. Scale bar: $5 \mu \mathrm{m}$. 
Figs. $5 \mathrm{a}$ and $5 \mathrm{~b}$ show the distributions of $\mathrm{k}_{\mathrm{a}}$ and $\mathrm{k}_{\mathrm{d}}$ values, respectively. The data were obtained by fitting the sensorgram of each bacterial cell (33 bacteria cells) with the first order binding kinetics model. Although the number of bacterial cells was limited, the data shows 2 orders of magnitude variability in $k_{a}$ and $k_{d} . K_{D}$ was obtained from the $k_{a}$ and $k_{d}$ values with the relation, $K_{D}=k_{d} / k_{a}$ (Fig. $5 c$ ). Unlike the distributions of $k_{a}$ and $k_{d}$, the distribution of $K_{D}$ has a center, which is near 3.9 $n M$. This $K_{D}$ value is close to the previously reported values in literature.(Medina et al., 1997) However, the presence of 4 orders of magnitude in $K_{D}$ conveys the heterogeneity in how an antibody might interact with individual cells.
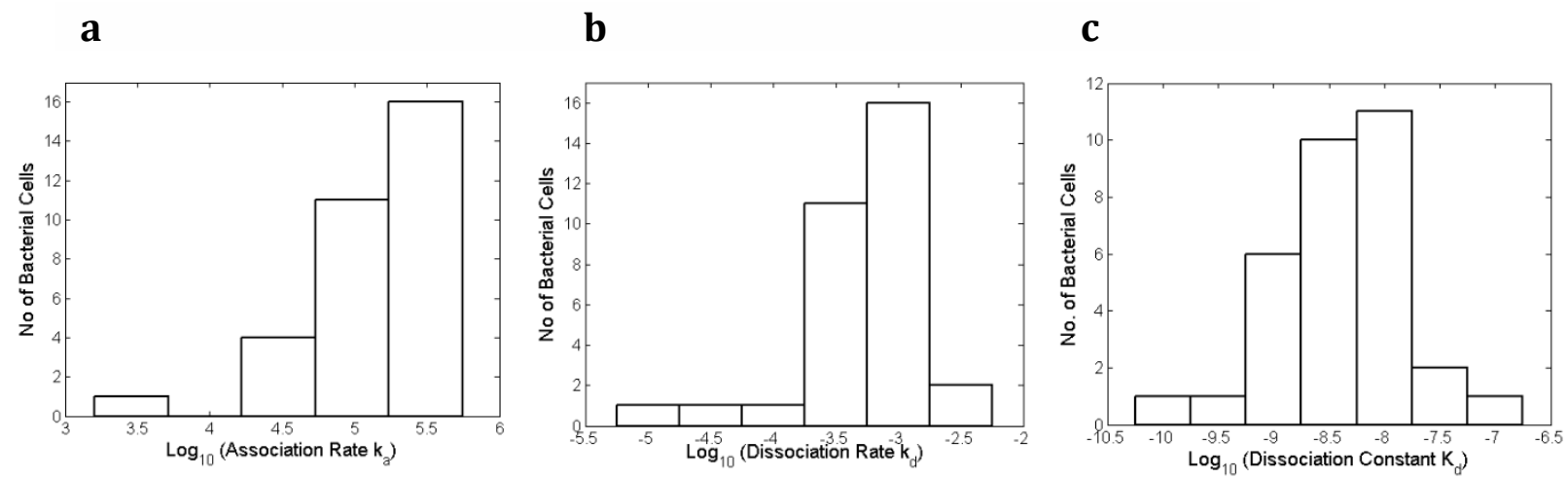

Fig. 5 Distributions of association rate, $\mathrm{k}_{\mathrm{a}}(\mathrm{a})$, dissociation rate, $\mathrm{k}_{\mathrm{d}}(\mathrm{b})$, dissociation constant, $\mathrm{K}_{\mathrm{D}}$, (c) observed on various bacteria. The large variability in the observed kinetics conveying cell-cell heterogeneity in Ab157 interacting with bacterial cells.

We also obtained distribution (Supplemental Fig. 2) of the plasmonic image intensity measured at the end of association phase from various bacterial cells. Since the plasmonic intensity is proportional to the mass density change of the sensor 
surface, the data reflects the distribution in the expression levels of $\mathrm{O}$-antigen on the outer membranes of the bacterial cells. We performed statistical analysis and found no obvious correlations between the measured 0 -antigen expression levels, $k_{a}$, $k_{d}$ and $K_{D}$ and the physical parameters, including length and size, of the bacteria (Supplemental Information - Table 1).

We believe that the reason for the observed cell-to-cell variability is the 0antigen on the surfaces of the bacteria,(Park et al., 1998) which is the target for the antibody. Studies have shown large variations in the chain length of 0 -antigen, which give E. Coli distinct surface morphologies (smooth, semi-rough and rough).(Reyes et al., 2012) This variation in chain length exists owing to Wzz and Wzy proteins, which are responsible for modulating the 0-antigen chain lengths. Wzz assembles the 0 -antigen around a specific modal length whereas Wzy assembles 0-antigen to a stochastic length.(Whitfield and Larue, 2008; Woodward et al., 2010) These variations in 0-antigen may be the primary reason for the observed cell-to-cell variability in the antibody binding kinetics.

The wide distributions in kinetic constants indicate the natural phenotypic diversity in a bacterial population. Capturing this diversity is important, especially because microbial sub-populations with variable phenotypic characteristics are known to play an important role in microbial evolution and antibiotic resistance.(Lidstrom and Konopka, 2010) The traditional bulk assays that measure average $K_{D}$ of a large number of bacteria cells could be misleading, especially when a species evolves and sub-populations emerge. In contrast, the present plasmonic imaging method can provide quantitative analysis of ligand interactions with 
individual live bacteria, which represents an unprecedented capability for studying the role of physiological heterogeneity in microbial population behaviors and providing new insights into microbial diversity arising from the rapid replication of bacteria.

\section{Conclusion}

We have described a plasmonic imaging method for studying the interaction kinetics between biomolecules and individual bacterial cells. The method is labelfree, quantitative and in real time. Using the method, we have measured the kinetics of an antibody binding to single E. Coli 0157:H7 bacterial cells. The mean values of the measured kinetic constants (e.g., $\mathrm{K}_{\mathrm{D}}$ ) are consistent with the reported literature values obtained from bulk assays. However, the present method revealed large cellto-cell variations in binding kinetics with kinetic constants distributed over several orders of magnitude. These results are direct evidence that large heterogeneity in the binding capability of bacteria with external ligands naturally exists in a bacterial population. Such heterogeneity has been hypothesized as an important mechanism for evolution and fitness in microbes. We anticipate that this method will improve the understanding of bacterial behaviors, such as pathogenesis and immune escape of virulent microbes, action and efficacy of antimicrobial peptides in acting on a microbial population and binding affinities of humanized antibodies against microbes for future drug therapies. 


\section{Acknowledgements}

The authors thank the financial support from (NSF Grant -1151005, CNSF Grant213270008).

\section{Supporting Information Available}

Results of negative control experiment, distribution of peak plasmonic image intensity after association phase and correlation of kinetics parameters can be found in the Supporting Information. This information can be found free of charge via the internet at 
References

Besser, R.E., Griffin, P.M., Slutsker, L., 1999. Annu. Rev. Med. 50, 355-67.

Carnes, E.C., Lopez, D.M., Donegan, N.P., Cheung, A., Gresham, H., Timmins, G.S., Brinker, C.J., 2010. Nat. Chem. Biol. 6, 41-5.

Casadevall, A., Dadachova, E., Pirofski, L., 2004. Nat. Rev. Microbiol. 2, 695-703.

Chang, P. V, Bertozzi, C.R., 2012. Chem. Commun. (Camb). 48, 8864-79.

Chiang, Y.-L., Lin, C.-H., Yen, M.-Y., Su, Y.-D., Chen, S.-J., Chen, H.-F., 2009. Biosens. Bioelectron. 24, 1905-10.

Cywes-Bentley, C., Skurnik, D., Zaidi, T., Roux, D., Deoliveira, R.B., Garrett, W.S., Lu, X., O’Malley, J., Kinzel, K., Zaidi, T., Rey, A., Perrin, C., Fichorova, R.N., Kayatani, A.K.K., Maira-Litràn, T., Gening, M.L., Tsvetkov, Y.E., Nifantiev, N.E., Bakaletz, L.O., Pelton, S.I., Golenbock, D.T., Pier, G.B., 2013. Proc. Natl. Acad. Sci. U. S. A. 110, E2209-18.

Delcour, A.H., 2009. Biochim. Biophys. Acta 1794, 808-16.

Fantner, G.E., Barbero, R.J., Gray, D.S., Belcher, A.M., 2010. Nat. Nanotechnol. 5, 2805.

Früh, V., IJzerman, A.P., Siegal, G., 2011. Chem. Rev. 111, 640-56.

Grant, C.F., Kanda, V., Yu, H., Bundle, D.R., McDermott, M.T., 2008. Langmuir 24, 14125-32.

Hirst, D.J., Lee, T.-H., Swann, M.J., Aguilar, M.-I., 2013. Anal. Chem. 85, 9296-304.

Holden, M. a, Jayasinghe, L., Daltrop, O., Mason, A., Bayley, H., 2006. Nat. Chem. Biol. 2, 314-8.

Huang, B., Yu, F., Zare, R.N., 2007. Anal. Chem. 79, 2979-83.

Jorgensen, J.H., Ferraro, M.J., 2009. Clin. Infect. Dis. 49, 1749-55.

Lee, A.G., 2004. Biochim. Biophys. Acta 1666, 62-87.

Lidstrom, M.E., Konopka, M.C., 2010. Nat. Chem. Biol. 6, 705-12. 
Liu, Q., Boyd, B.J., 2013. Analyst 138, 391-409.

Longo, G., Alonso-Sarduy, L., Rio, L.M., Bizzini, a, Trampuz, a, Notz, J., Dietler, G., Kasas, S., 2013. Nat. Nanotechnol. 8, 522-6.

Lower, S.K., Lamlertthon, S., Casillas-Ituarte, N.N., Lins, R.D., Yongsunthon, R., Taylor, E.S., DiBartola, A.C., Edmonson, C., McIntyre, L.M., Reller, L.B., Que, Y.-A., Ros, R., Lower, B.H., Fowler, V.G., 2011. Proc. Natl. Acad. Sci. U. S. A. 108, 18372-7.

Medina, M.B., Houten, L. Van, Cooke, P.H., Tu, S.I., 1997. Biotechnol Tech. 11, 173176.

Park, C.H., Martin, E. a, White, E.L., 1998. J. Clin. Microbiol. 36, 1408-9.

Reyes, R.E., González, C.R., Jiménez, R.C., Herrera, M.O., Andrade, A.A., 2012. doi:10.5772/48147

Shan, X., Díez-Pérez, I., Wang, L., Wiktor, P., Gu, Y., Zhang, L., Wang, W., Lu, J., Wang, S., Gong, Q., Li, J., Tao, N., 2012. Nat. Nanotechnol. 7, 668-72.

Shen, Z., Huang, M., Xiao, C., Zhang, Y., Zeng, X., Wang, P.G., 2007. Anal. Chem. 79, 2312-2319.

Sochacki, K. a, Barns, K.J., Bucki, R., Weisshaar, J.C., 2011. Proc. Natl. Acad. Sci. U. S. A. 108, E77-81.

Subramanian, A., Irudayaraj, J., Ryan, T., 2006. Biosens. Bioelectron. 21, 998-1006.

Tawil, N., Sacher, E., Mandeville, R., Meunier, M., 2012. Biosens. Bioelectron. 37, 249.

Torun, O., Hakkı Boyacı, I., Temür, E., Tamer, U., 2012. Biosens. Bioelectron. 37, 5360.

Tracy, B.P., Gaida, S.M., Papoutsakis, E.T., 2010. Curr. Opin. Biotechnol. 21, 85-99.

Van der Mei, H.C., Busscher, H.J., 2012. PLoS Pathog. 8, e1002821.

Van Houdt, R., Michiels, C.W., 2005. Res. Microbiol. 156, 626-33.

Wang, S., Shan, X., Patel, U., Huang, X., Lu, J., Li, J., Tao, N., 2010. Proc. Natl. Acad. Sci. U. S. A. 107, 16028-32.

Wang, W., Foley, K., Shan, X., Wang, S., Eaton, S., Nagaraj, V.J., Wiktor, P., Patel, U., Tao, N., 2011. Nat. Chem. 3, 249-55. 
Wang, W., Yang, Y., Wang, S., Nagaraj, V.J., Liu, Q., Wu, J., Tao, N., 2012. Nat. Chem., 4, 846-53.

Whitfield, C., Larue, K., 2008. Nat. Struct. Mol. Biol. 15, 121-3.

Woodward, R., Yi, W., Li, L., Zhao, G., Eguchi, H., Sridhar, P.R., Guo, H., Song, J.K., Motari, E., Cai, L., Kelleher, P., Liu, X., Han, W., Zhang, W., Ding, Y., Li, M., Wang, P.G., 2010. Nat. Chem. Biol. 6, 418-23.

Woude, M.W. Van Der, Bäumler, A.J., 2004. Clin. Microbiol. Rev. 581-611

Zordan, M.D., Grafton, M.M.G., Acharya, G., Reece, L.M., Cooper, C.L., Aronson, A.I., Park, K., Leary, J.F., 2009. Cytometry. A 75, 155-62. 